\title{
EGU21-8012
}

https://doi.org/10.5194/egusphere-egu21-8012

EGU General Assembly 2021

(c) Author(s) 2021. This work is distributed under

the Creative Commons Attribution 4.0 License.

\section{Evaluating nature-based solutions in a non-stationary climate with changing risk of flooding}

\author{
Sisay Debele ${ }^{1}$, Jeetendra Sahani ${ }^{1}$, Silvia Maria Alfieri ${ }^{3}$, Paul Bowyer ${ }^{4}$, Nikos Charizopoulos ${ }^{5,6}$, \\ Michael Loupis ${ }^{7,8}$, Massimo Menenti, ${ }^{3,9}$, Fabrice Renaud ${ }^{10}$, Mohammad Aminur Rahman Shah ${ }^{10}$, \\ Christos Spyrou ${ }^{7,11}$, Thomas Zieher ${ }^{12}$, Silvana Di Sabatino ${ }^{13}$, and Prashant Kumar ${ }^{1,2}$ \\ ${ }^{1}$ Global Centre for Clean Air Research (GCARE), Department of Civil and Environmental Engineering, Faculty of Engineering \\ and Physical Sciences, University of Surrey, Guildford GU2 7XH, United Kingdom \\ ${ }^{2}$ Department of Civil, Structural and Environmental Engineering, School of Engineering, Trinity College Dublin, Dublin, \\ Ireland \\ ${ }^{3}$ Department of Geoscience and Remote Sensing, Delft University of Technology, Delft, The Netherlands \\ ${ }^{4}$ Climate Service Center Germany (GERICS), Helmholtz-Zentrum Geesthacht, Hamburg, Germany \\ ${ }^{5}$ Agricultural University of Athens, Laboratory of Mineralogy-Geology, lera Odos 75, 11855 Athens, Greece \\ ${ }^{6}$ Region of Sterea Ellada, Kalivion 2, 351 32, Lamia, Greece \\ ${ }^{7}$ Innovative Technologies Center S.A., Alketou Str. 25, 11633 Athens, Greece \\ ${ }^{8}$ National \& Kapodistrian University of Athens, Psachna 34400, Greece \\ ${ }^{9}$ Aerospace Information Research Institute, Chinese Academy of Sciences, Beijing, China \\ ${ }^{10}$ School of Interdisciplinary Studies, University of Glasgow, Dumfries Campus, DG1 4ZL, United Kingdom \\ ${ }^{11}$ Institute for Astronomy, Astrophysics, Space Applications and Remote Sensing (IAASARS), National Observatory of \\ Athens, 15236 Athens, Greece \\ ${ }^{12}$ Institute for Interdisciplinary Mountain Research, Austrian Academy of Sciences, Technikerstr. 21a, 6020 Innsbruck \\ Austria \\ ${ }^{13}$ Department of Physics and Astronomy (DIFA), University of Bologna, Bologna, Italy
}

\begin{abstract}
Under climate change scenarios, it is important to evaluate the changes in recent behavior of heavy precipitation events, the resulting flood risk, and the detrimental impacts of the peak flow of water on human well-being, properties, infrastructure, and the natural environment. Normally, flood risk is estimated using the stationary flood frequency analysis technique. However, a site's hydroclimate can shift beyond the range of historical observations considering continuing global warming. Therefore, flood-like distributions capable of accounting for changes in the parameters over time should be considered. The main objective of this study is to apply non-stationary flood frequency models using the generalized extreme value (GEV) distribution to model the changes in flood risk under two scenarios: (1) without nature-based solutions (NBS) in place and; (2) with NBS i.e. wetlands, retention ponds and weir/low head dam implemented. In the GEV model, the first two moments i.e. location and scale parameters of the distribution were allowed to change as a function of time-variable covariates, estimated by maximum likelihood. The methodology is applied to OPEn-air laboRAtories for Nature baseD solUtions to Manage hydro-meteo risks, which
\end{abstract}


is in Europe. The time-dependent 100-year design quantiles were estimated for both the scenarios. We obtained daily precipitation data of climate models from the EURO-CORDEX project dataset for 1951-2020 and 2022-2100 representing historical and future simulations, respectively. The hydrologic model, HEC-HMS was used to simulate discharges/flood hydrograph without and with NBS in place for these two periods: historical (1951-2020) and future (2022-2100). The results showed that the corresponding time-dependent 100-year floods were remarkably high for the without NBS scenario in both the periods. Particularly, the high emission scenario (RCP 8.5) resulted in dramatically increased flood risks in the future. The simulation without NBS also showed that flooded area is projected to increase by $25 \%$ and $40 \%$ for inundation depth between 1.5 and 3.5 m under RCP 4.5 and RCP 8.5 scenarios, respectively. For inundation depth above 3.5 $\mathrm{m}$, the flooded area is anticipated to rise by $30 \%$ and $55 \%$ in both periods respectively. With the implementation of NBS, the flood risk was projected to decrease by $20 \%$ (2022-2050) and $45 \%$ (2071-2100) with a significant decrease under RCP 4.5 and RCP 8.5 scenarios. This study can help improve existing methods to adapt to the uncertainties in a changing environment, which is critical to develop climate-proof NBS and improve NBS planning, implementation, and effectiveness assessment.

Keywords: Nature-based solutions; flood frequency analysis; climate change; wetlands; GEV model

\section{Acknowledgments}

This work has been carried out under the framework of OPERANDUM (OPEn-air laboRAtories for Nature baseD solUtions to Manage hydro-meteo risks) project, which is funded by the European Union's Horizon 2020 research and innovation programme under the Grant Agreement No: 776848. 